\title{
Theory of Josephson Phenomena in Superfluid ${ }^{3} \mathrm{He}$
}

\author{
Erkki Thuneberg
}

Department of Physical Sciences, University of Oulu, Finland

\begin{abstract}
Quite detailed theoretical description of superfluid ${ }^{3} \mathrm{He}$ is possible on length scales that are much larger than the atomic scale. We discuss weak links between two bulk states of ${ }^{3} \mathrm{He}-\mathrm{B}$. The current through the weak link is determined by the bound states at the link. The bound state energies are spin split depending on the order parameters in the bulk. As a result, unusual current-phase relations with $\pi$ state appear. For not too weak links, the order parameter in the bulk is modified because of the Josephson coupling. This leads to a stronger $\pi$ state and to an additional current at constant pressure bias. The theoretical results are compared with experiments.
\end{abstract}

\section{INTRODUCTION}

In spite of more than thirty years of studies of superfluid phases of ${ }^{3} \mathrm{He}$, there are several active research directions. One of them is the study of Josephson phenomena in weak links between two volumes of bulk superfluid. Here we give a review of recent theoretical work on this subject [1, 2, 3, 4]. We pay special attention to the role of bound quasiparticle states in the link, and explain how these determine the flow through the junction. In particular we study the equilibrium current-phase relation, $I(\phi)$, and the dc current at constant potential difference, $I_{\mathrm{dc}}(U)$. We compare the results with experiments. The experimental work has been reviewed in Ref. [5]. A theoretical review with a somewhat different emphasis has been given in Ref. [6].

\section{WEAK LINKS}

Let us consider two volumes of superfluid that are connected by a weak link. Here "superfluid" means generally a fermion superfluid, either superconducting metal or liquid ${ }^{3} \mathrm{He}$. Our discussion of superconducting weak links is mainly aimed as an introduction for ${ }^{3} \mathrm{He}$. Therefore we neglect all complications that arise from impurity or interfacial scattering in superconductors. Also we consider only superconductors of the conventional (s-wave) type. In the case of ${ }^{3} \mathrm{He}$ we limit to the superfluid B phase.

The geometry of the weak link is depicted in Fig. 1. The figure shows one quasiparticle trajectory with momentum p. In bulk superfluid such elementary excitations must have energy $\varepsilon$ larger than the energy gap $\Delta$ of the superfluid. In the weak link, however, there exists en-

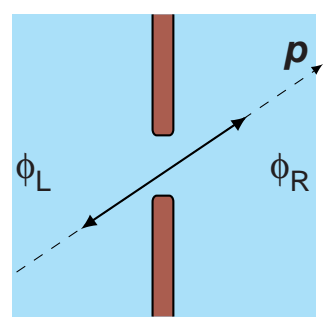

FIGURE 1. Sketch of a weak link between two bulk superfluids. The two bulk superfluids have phases $\phi_{L}$ and $\phi_{R}$. One quasiparticle trajectory with momentum $\mathbf{p}$ is shown. When its energy is within the energy gap, the quasiparticle cannot escape to the bulk but is Andreev reflected as hole with essentially the same momentum. This in turn is Andreev reflected as particle on the other side of the link. Thus particle and hole type excitations bounce back and forth along the same trajectory.

ergy eigenvalues within the energy gap, $|\varepsilon|<\Delta$. In such a state the quasiparticle cannot escape to the bulk but is Andreev reflected. In Andreev reflection a particle type excitation is converted to hole type and vice versa, with essentially unchanged momentum [7]. Thus the particles and holes travel the same trajectory back and forth, respectively. In one cycle, one Cooper pair, or equivalently two particles, are transmitted through the weak link.

The bulk superfluid states are characterized by phases $\phi_{L}$ and $\phi_{R}$. The energies of the bound states depend essentially on the phase difference $\phi=\phi_{R}-\phi_{L}$ [8]. For superconductors this is depicted in Fig. 2] The states appear as pairs with positive and negative energies, $\pm|\varepsilon|$. Understanding that $d \varepsilon / d \phi$ plays a similar role as the group velocity, we can assign the states as propagating to the right $(\delta=+1)$ and to the left $(\delta=-1)$. These two states are not independent, however. Based on what was stated about Andreev reflection above, if a state with $\delta=$ 


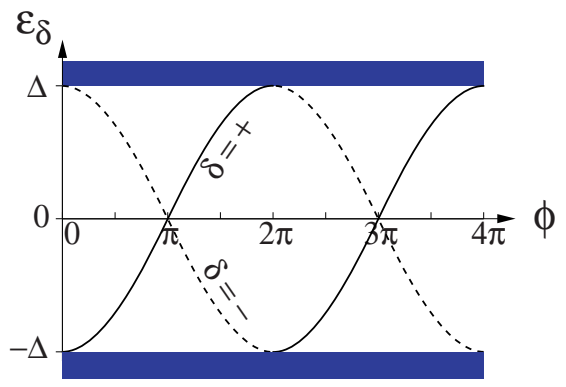

FIGURE 2. Bound state energies $\varepsilon_{\delta}(\phi)$ in a superconducting point contact. As $\phi$ increases, the states with transport to the right $(\delta=+1)$ have increasing energy (solid lines), and the states with transport to the left $(\delta=-1)$ have decreasing energy (dashed lines). The states at energies $|\varepsilon|>\Delta$ form a continuum.

+1 is occupied, the corresponding $\delta=-1$ state must be empty. And vice versa. More generally, the occupations $f_{ \pm}$have to satisfy $f_{+}+f_{-}=1$. Really the states with positive and negative energies are the same bound state, whose energy relative to the ground state is the absolute value $|\varepsilon|$. Anyhow, we find it very useful to consider a bound state as a superposition of positive and negative energy states.

In the simplest case of a point contact, the bound state energies have the simple analytic expression $\varepsilon=$ $\pm \Delta \cos \frac{\phi}{2}[8]$. By a point contact, or a pinhole in case of ${ }^{3} \mathrm{He}$, we mean a weak link whose all dimensions are small compared to the superfluid coherence length $\xi_{0}$.

It is an interesting observation that to a large extent the current through a weak link is determined by the bound states [9, 10, 11]. In the following we are interested in equilibrium or only small nonequilibrium, where the chemical potential difference $\mu_{L}-\mu_{R} \equiv U \ll \Delta \sim k_{B} T_{c}$. In this case the current has the form

$$
I=\frac{2}{\hbar} \sum_{i} \sum_{\delta} \frac{d \varepsilon_{i \delta}}{d \phi} f_{i \delta}
$$

Here $f_{i \delta}$ is the occupation of the state. In equilibrium it reduces to the Fermi distribution $f_{i \delta}=\left[\exp \left(\varepsilon_{i \delta} / k_{B} T\right)+\right.$ $1]^{-1}$. The index $i$ indicates different channels, which correspond to different directions and locations of the bound state (Fig. 11). In a point contact of area $A$, there are $M=k_{F}^{2} A / 4 \pi$ channels, where $k_{F}$ is the Fermi wave vector. In the normal state the current is $I=M U / \pi \hbar$ [12].

Kulik and Omel'yanchuk derived in 1975 the following formula for equilibrium current trough a superconducting point contact [13]

$$
I=\frac{M \Delta}{\hbar} \sin \frac{\phi}{2} \tanh \frac{\Delta \cos \frac{\phi}{2}}{2 k_{B} T} .
$$

Their original derivation does not give much clue to understand the result. Now this result can be straightforwardly understood based on the formulas given above:

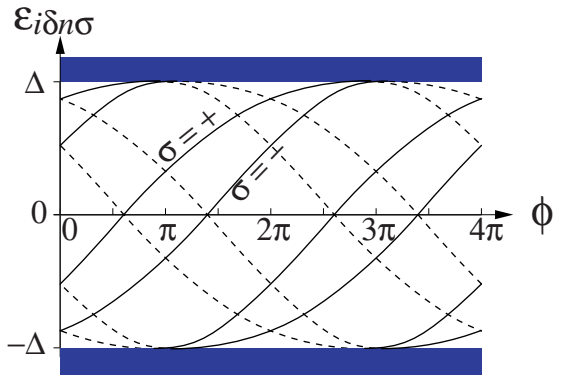

FIGURE 3. Example of bound states energies $\varepsilon_{i \delta n \sigma}(\phi)$ in a ${ }^{3} \mathrm{He}$ weak link. Compared to Figure 2 the bound state energies have smaller slopes and they are spin split $(\sigma= \pm 1)$. Figure reprinted from Ref. [3].

all channels have the same bound state energies $\varepsilon=$ $\pm \Delta \cos \frac{\phi}{2}$ and using the current formula (1) and the Fermi distribution gives the result (2).

As the temperature increases towards the superfluid transition temperature $T_{c}$, the Kulik-Omel'yanchuk result (2) reduces to sinusoidal form

$$
I=I_{c} \sin \phi
$$

with critical current $I_{c}=M \Delta^{2} / 4 \hbar k_{B} T_{c}$.

\section{${ }^{3}$ HE WEAK LINKS}

Most of the discussion of superconducting weak links applies also to weak links in ${ }^{3} \mathrm{He}$. There are a few differences. In ${ }^{3} \mathrm{He}$ the Cooper pairs are in p-wave states, instead of the s-wave state considered above. Any scattering breaks these pairs. Thus the superfluid state is always suppressed near walls. This suppression also affects the bound states energies in a weak link, as depicted in Fig. 3 Compared to the superconducting case, the slopes $d \varepsilon / d \phi$ are smaller in magnitude. Another difference is that there is spin splitting of the energy states $(\sigma= \pm 1)$. Whereas the states in the superconducting case were doubly degenerate, this degeneracy is generally broken in ${ }^{3} \mathrm{He}$.

Because of the spin splitting the current formula (1) has to be generalized to the form

$$
I=\frac{1}{\hbar} \sum_{i} \sum_{\delta} \sum_{n} \sum_{\sigma} \frac{d \varepsilon_{i \delta n \sigma}}{d \phi} f_{i \delta n \sigma}
$$

where a factor of 2 is replaced by spin summation. There is also additional summation over index $n$, which takes into account that several bound states can occur at a given $\phi$.

The spin splitting can lead to current-phase relationships that differ essentially from the standard sinusoidal 


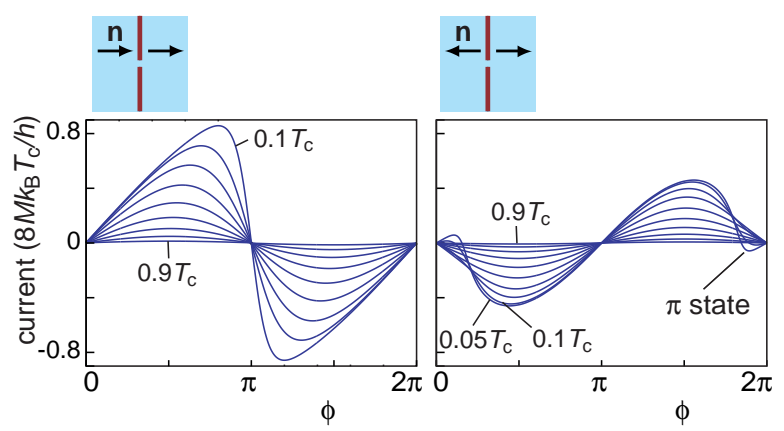

FIGURE 4. Current-phase relationships calculated for a pinhole. The left hand panel is for parallel $\hat{\mathbf{n}}$ vectors on the two sides of the junction. The right hand panel is for antiparallel $\hat{\mathbf{n}}$ 's that are perpendicular to the wall. At high temperature the curves are sinusoidal (3). At lower temperature the curves become skew in the parallel case resembling the KulikOmel'yanchuk result (2). The antiparallel case has negative critical current $I_{c}$, or equivalently, is shifted by a phase difference $\pi$. At very low temperature it develops an additional kink that is known as $\pi$ state. Figure adapted from Ref. [2].

form (3) [14]. Consider, for example, the case where the spin split states are shifted relative to each other by a phase difference $\approx \pi$. It follows that the leading sinusoidal components of the current from the two spin states $\left[\propto \sin \left(\phi+\psi_{\sigma}\right)\right]$ cancel each other. What is then left are higher harmonics $\left[\propto \sin \left(n \phi+\psi_{\sigma}\right), n=3, \ldots\right]$. Unusual current-phase relations are confirmed by calculations below.

The Cooper pairs of superfluid ${ }^{3} \mathrm{He}$ have $\mathrm{p}$-wave form [15]. There are three orthogonal p-wave states: $p_{x}, p_{y}$, and $p_{z}$. The spin state is triplet. There are three triplet states, which conventionally are chosen as $-\uparrow \uparrow+\downarrow \downarrow$, $i \uparrow \uparrow+i \downarrow \downarrow$, and $-\uparrow \downarrow+\downarrow \uparrow$. Here we concentrate on the B-phase, where $p_{x}$ pairs have the first spin state, $p_{y}$ pairs the second and $p_{z}$ pairs the third. However, the spin coordinate axes $\left(x^{\prime}, y^{\prime}, z^{\prime}\right)$ are rotated relative to the orbital axes $(x, y, z)$. The rotation angle is fixed to $104^{\circ}$, but the axis $\hat{\mathbf{n}}$ of this rotation can vary.

In hydrodynamics of the B phase the mass $(\phi)$ and spin $(\hat{\mathbf{n}})$ degrees of freedom are independent. The weak link acts as a nonlinear element that couples mass and spin, as will be demonstrated below.

\section{Isotextural Theory}

Some current-phase relations calculated for a ${ }^{3} \mathrm{He}-\mathrm{B}$ pinhole are shown in Fig. 4 Two cases are shown. First, the spin rotation axes $\hat{\mathbf{n}}$ on both sided of the junction are the same, and second, the rotation axes have opposite directions perpendicular to the wall of the pinhole. The former case resembles the Kulik-Omel'yanchuk result (2): at high temperatures $I(\phi)$ is sinusoidal but at lower tem-

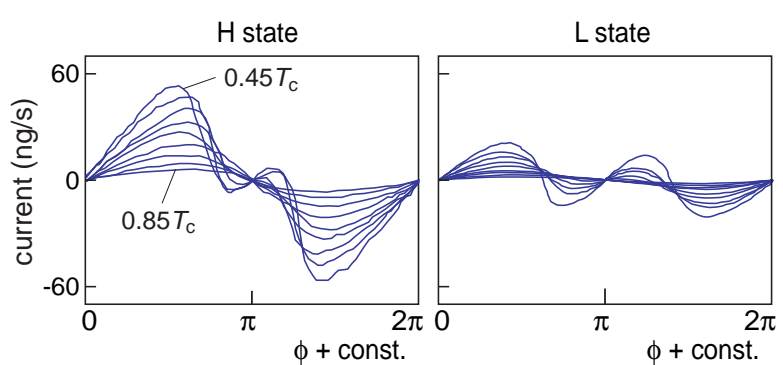

FIGURE 5. Measured current-phase relationships for a $65 \times$ 65 array of $100 \mathrm{~nm}$ diameter apertures in $50 \mathrm{~nm}$ thick wall [16]. In cooling through $T_{\mathrm{c}}$, the system randomly freezes to either $\mathrm{H}$ or L state. Only the relative value of the phase is determined experimentally. Figure adapted from Ref. [16].

peratures it becomes skew towards $\phi=\pi$. The case with antiparallel $\hat{\mathbf{n}}$ 's is also sinusoidal at high temperatures, but has negative $I_{c}$. (Such a case is called a $\pi$ junction in superconductivity.) At temperatures around $0.1 T_{c}$ it develops to a $\pi$ state. This means that the slope of $I(\phi)$ is positive at both 0 and $\pi$.

Prior theoretical calculations, the $\pi$ state had been observed experimentally. The experimental results of Ref. [16] are shown in Fig. 5 The experimental $I(\phi)$ is sinusoidal at high temperature but develops a $\pi$ state at low temperature. Moreover, two different metastable states with different sets of $I(\phi, T)$ curves was observed. These are called $\mathrm{H}$ and $\mathrm{L}$, corresponding to high and low critical current. The two states appeared randomly in cooling through $T_{c}$, but the state selected remained stable during the stay in the superfluid state.

Let us consider a weak link under a constant chemical potential difference $U=\mu_{L}-\mu_{R}$. According to the Josephson relation

$$
\frac{d \phi}{d t}=\frac{2 U}{\hbar}
$$

the phase $\phi$ increases linearly in time. This implies that the bound state energies (Fig. 3) are continuously shifting up or down. During shifting the occupations of these states can change in collisions with other quasiparticles. Since the collisions are rare, this thermalizes the occupations only at small $U<\hbar / \tau$, where $\tau$ is the scattering time. At larger bias, the occupations of the bound states remain essentially constant during the shift from $-\Delta$ to $+\Delta$, or from $+\Delta$ to $-\Delta$. The occupations of these states are then fixed to the thermal equilibrium occupations at the starting energies, $-\Delta$ or $+\Delta$, respectively.

It is now possible to calculate the current using Eq. (4). The resulting time-averaged current $I_{\mathrm{dc}}$ is plotted in Fig. 6 At large bias $(U \gg \hbar / \tau)$ the occupation are determined by gap edges and the current therefore becomes independent of the bias $U$. At small bias $(U \ll \hbar / \tau)$ the scattering has time to preserve a nearly thermal distribu- 


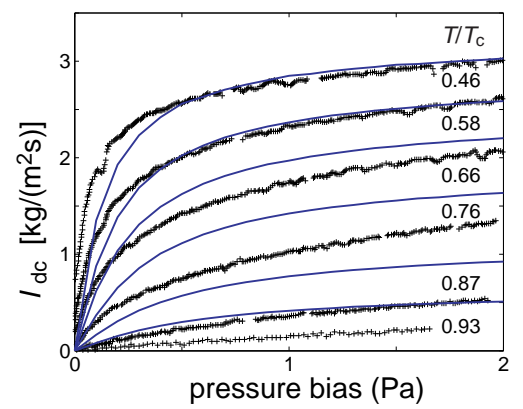

FIGURE 6. Average current vs. pressure bias. The data points are experimental results from Ref. [17], and the lines are theoretical results from Ref. [4]. The pressure bias of $1 \mathrm{~Pa}$ corresponds to $U=4.7 \times 10^{-3} k_{B} T_{c}$. Figure reprinted from Ref. [4].

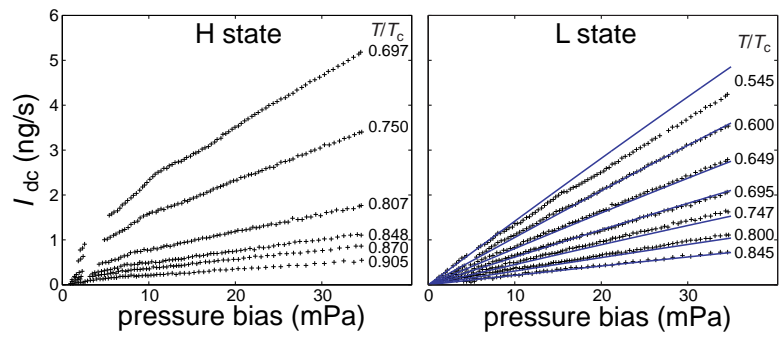

FIGURE 7. Average current vs. pressure at low pressure bias. The data points are experimental results from Ref. [18] for the same $65 \times 65$ array of apertures as the equilibrium currents in Fig. 5] The lines drawn on the right hand side panel are the results of isotextural theory, and fitted with a single parameter (the relaxation time) as in Fig. 6 According to isotextural theory, the same lines should fit also the $\mathrm{H}$ state, which clearly is not the case. Figure adapted from Ref. [3].

tion, and the deviation from equilibrium distribution as well as the current is linear in $U$. Note that the equilibrium current (Fig. (4) is oscillating, and therefore does not contribute to the time-averaged current.

Fig. 6 shows also experimental data by Steinhauer et al. [17]. The theory can reasonably be fitted to this data using the scattering time as the only fitting parameter. The agreement is surprisingly good taking into account that the single aperture in the experiment (dimensions $7.8 \mu \mathrm{m} \times 0.27 \mu \mathrm{m}$ ) is large compared to the superfluid coherence length $\xi_{0}=77 \mathrm{~nm}$, whereas the pinhole theory makes just the opposite assumption.

The current at finite bias was also measured for the same array of apertures as the equilibrium current in Fig. [5 The results are shown in Fig. [7 The current is different for the $\mathrm{H}$ and $\mathrm{L}$ states. The current in the L state is in reasonable agreement with theory using again the relaxation time as the only adjustable parameter.

As a summary thus far, we can say that theory and experiment are in reasonable agreement. Both show bista- bility ( $\mathrm{H}$ and $\mathrm{L}$ state), $\pi$ states, and similar dissipative currents $I_{\mathrm{dc}}(U)$. The temperature dependencies agree as well the order of magnitudes of all quantities.

In spite of this success, closer examination reveals some problems in the theory. 1) Only one of theoretical bistable states shows $\pi$ state (Fig. 4). More detailed analysis reveals that this problem cannot be removed by considering other configurations of $\hat{\mathbf{n}}$ 's [2]. 2) The theoretical $\pi$ state occurs at a much lower temperature and is much weaker than in experiments (compare Figs. 4 and 55. 3) The theory does not predict any difference in $I_{\mathrm{dc}}(U)$ between the $\mathrm{H}$ and $\mathrm{L}$ states. This is because different $\hat{\mathbf{n}}$ configurations mainly shift the bound states energies $\varepsilon_{i \delta n \sigma}$ along the $\phi$ axis but do not much affect the shape of the $\varepsilon_{i \delta n \sigma}(\phi)$ curves. In running $\phi$ this only affects the instantaneous current $I(t)$, but the average $I_{\mathrm{dc}}$ is unchanged.

\section{Anisotextural Theory}

All the problems above can be explained by a single new concept. If the Josephson coupling is strong, it can change the spin-part of the order parameter on both sides. We call this anisotextural effect, since the $\hat{\mathbf{n}}$ orientations are commonly known as texture.

As an example consider the case of parallel $\hat{\mathbf{n}}$ 's. Then there is no spin splitting and the bound state at phase difference $\phi=\pi$ lies at zero energy, $\varepsilon=0$. The thermal occupation of this doubly degenerate state is $f=1 / 2$. If we now allow spin splitting, the $\varepsilon=0$ state can split into two with positive and negative energies (Fig. 3). Taking into account that the occupation of the negative energy state is larger than that of the positive energy state, this leads to a reduction of energy. Thus the spin splitting can take place spontaneously.

The anisotextural effect as a function of phase is shown in Fig. 8 Starting from the completely spinsymmetric situation at $\phi=0$, the $\hat{\mathbf{n}}$ texture changes spontaneously as $\phi$ increases towards $\pi$. The reduction of the Josephson coupling energy $F_{\mathrm{J}}$ is associated with a change in $I(\phi)$ since

$$
I=\frac{2}{\hbar} \frac{\partial F_{\mathrm{J}}}{\partial \phi}
$$

Thus a $\pi$ state develops if the reduction of $F_{\mathrm{J}}$ is sufficient to produces a local minimum of $F_{\mathrm{J}}(\phi)$ at $\phi=\pi$.

An additional contribution to energy arises from the bending of $\hat{\mathbf{n}}$ (right hand panel of Fig. 8). In a simple model the total energy is written as

$$
F[\eta]=F_{J}\left(\eta_{0}, \phi\right)+\frac{1}{2} K \int d^{3} r|\nabla \eta|^{2} .
$$

The first term is the Josephson coupling energy that depends on $\phi$ and on the tilting angle $\eta_{0}$ of $\hat{\mathbf{n}}$ at the weak link. The second term is a gradient energy for the tilting 


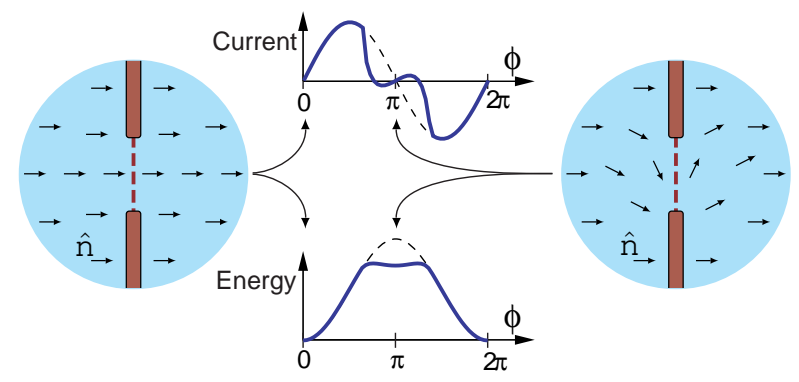

FIGURE 8. Principle of the anisotextural Josephson effect. The panels on the left and right hand sides depict an array of apertures and the configurations of the spin-rotation axis $\hat{\mathbf{n}}$. Assuming there is no spin structure $(\hat{\mathbf{n}}=$ constant $)$ at the phase difference $\phi=0$, the $\hat{\mathbf{n}}$ texture changes spontaneously when $\phi$ increases to $\pi$. This leads to a reduction of energy and to a positive slope of $I(\phi)$ at $\phi=\pi$, as shown by the change from dashed lines to solid lines in the current and energy plots.

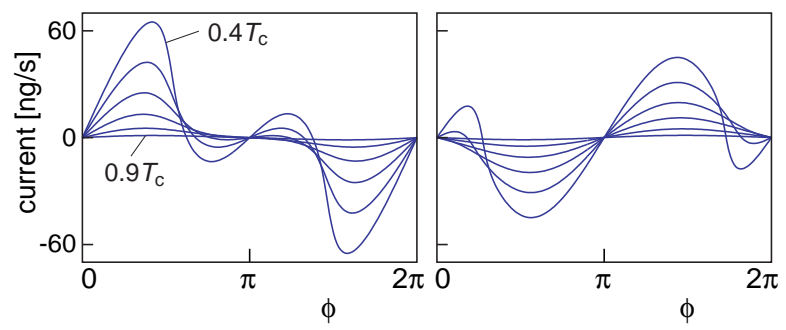

FIGURE 9. Current-phase relationships calculated using the anisotextural model (7). The theory contains one free parameter $\eta_{\infty}$, and the result should be compared with the experimental one in Fig. [5] Figure adapted from Ref. [2].

angle in the bulk. In comparison to experiments, the only free parameter is the tilting angle $\eta_{\infty}$ on one side far away from the junction, which is difficult to calculate because of the complicated shape of the experimental cell. Using the freedom to adjust $\eta_{\infty}$, it is possible to generate the current-phase relations shown in Fig. 9 This should be compared with the experimental curves in Fig. 5]

Next we consider the anisotextural effect at constant bias $U$. Finite $U$ means that the phase changes at constant rate [Eq. [5] ]. As a result of the anisotextural effect, the $\hat{\mathbf{n}}$ texture oscillates at the angular frequency $\omega=$ $2 U / \hbar$. This oscillation of the spin structure generates spin waves, which radiate out of the junction.

In order to make an quantitative theory, we need to add one more contribution to the energy (7) so that the total energy is

$$
\begin{array}{r}
F[\eta, S]=F_{J}\left(\eta_{0}, \phi\right)+\frac{1}{2} K \int d^{3} r|\nabla \eta|^{2} \\
+\frac{\gamma^{2}}{2 \chi} \int d^{3} r S^{2} .
\end{array}
$$

The last therm is the energy associated with net spin density $S$, where $\gamma$ is the gyromagnetic ratio and $\chi$ the magnetic susceptibility [19]. Here $S$ and $\eta$ are conjugate variables. Writing the Hamiltonian equations, this leads to a wave equation describing the spin waves and to a boundary condition for them. It turns out to be a quite standard radiation problem. The radiated power has the frequency dependence

$$
P_{\mathrm{rad}} \propto \frac{\omega^{2}}{1+\left(r_{0} \omega / c\right)^{2}},
$$

where $r_{0}$ is the radius of the Josephson array, and $c$ the spin wave velocity. This expresses that there is little radiation for wave lengths longer than the source, but more at shorter wave lengths.

The dissipated power now has to come from the $\mathrm{dc}$ current, $P=U I_{\mathrm{dc}}$. Thus there is additional dc current due to the spin wave radiation. Including also the prefactors we get for it the expression

$$
I_{\mathrm{dc}, \mathrm{rad}}=\frac{P_{\mathrm{rad}}}{U}=\frac{2}{\hbar} \frac{\left[J_{\mathrm{sp}}\left(\eta_{\infty}\right)\right]^{2}}{4 \pi c K} \frac{\omega}{1+\left(\omega r_{0} / c\right)^{2}} .
$$

Here the equilibrium spin current $J_{\mathrm{sp}}\left(\eta_{\infty}\right)$ plays a role of a coupling constant between mass and spin variables. Most interestingly, this coupling is different for the theoretical $\mathrm{H}$ and $\mathrm{L}$ states. Putting in numbers we see that the theory explains approximately one third of the observed H-L difference shown in Fig. 5 (The comparison is shown in Ref. [3].) This is not completely satisfactory, but we must remember that all parameters of the theory were fixed before the comparison.

Models to explain the observed $I_{\mathrm{dc}}(U)$ have also been suggested in Ref. [18]. These models differ essentially from the present ones. In particular, an A-phase-like distortion of the order parameter is associated with the linear part and a quasiparticle radiation mechanism with the nonlinear part of $I_{\mathrm{dc}}(U)$.

\section{CONCLUSION}

We have seen that coupling between mass and spin variables is essential for understanding the Josephson phenomena in superfluid ${ }^{3} \mathrm{He}$. With spin splitting of bound states together with the anisotextural effect it is possible to explain most of the experimental results of both $I(\phi)$ and $I_{\mathrm{dc}}(U)$. The theory presented above uses the pinhole approximation. For more accurate results it is necessary to consider larger apertures. This would require selfconsistent determination of the order parameter within the aperture, which is calculationally demanding and has been done only in limiting cases [20, 21]. We expect that this might lead to qualitatively same type of results as the anisotextural effect for pinhole arrays, in particular, to enhanced $\pi$ states and to a texture dependence 
of $I_{\mathrm{dc}}(U)$. This might explain the remaining differences between theory and experiment, and the experimental results in single apertures [22].

The anisotextural phenomena depend strongly on many parameters such as array size, geometry of the experimental cell and magnetic field. We hope that these could be tested in future experiments.

\section{REFERENCES}

1. J. K. Viljas and E. V. Thuneberg, Phys. Rev. Lett. 83, 3868 (1999).

2. J. K. Viljas and E. V. Thuneberg, Phys. Rev. B 65, 064530 (2002).

3. J. K. Viljas and E. V. Thuneberg, Phys. Rev. Lett. 93, 205301 (2004).

4. J. K. Viljas, Phys. Rev. B 71, 064509 (2005).

5. J. C. Davis and R. E. Packard, Rev. Mod. Phys. 74, 741 (2002).

6. J. K. Viljas and E. V. Thuneberg, J. Low Temp. Phys. 136, 329 (2004).

7. A.F. Andreev, J. Eksp. Teor. Fiz. 49, 655 (1965) [Sov. Phys. JETP 22, 455 (1966)].

8. I. O. Kulik, Zh. Eksp. Teor. Fiz. 57, 1745 (1969) [Sov. Phys. JETP 30, 944 (1970)].

9. A. Furusaki and M. Tsukada, Physica B 165\&166, 967 (1990).

10. C. W. J. Beenakker, Phys. Rev. Lett. 67, 3836 (1991).

11. D. Averin and A. Bardas, Phys. Rev. B 53, R1705 (1996).

12. S. Datta, Electronic Transport in Mesoscopic Systems (Cambridge, 1995).

13. I. O. Kulik and A. N. Omel'yanchuk, Fiz. Nizk. Temp. 3, 945 (1977) [Sov. J. Low Temp. Phys. 3, 459 (1977)].

14. S.-K. Yip, Phys. Rev. Lett. 83, 3864 (1999).

15. A.J. Leggett, Rev. Mod. Phys. 47, 331 (1975).

16. A. Marchenkov, R.W. Simmonds, S. Backhaus, K. Shokhirev, A. Loshak, J. C. Davis, and R. E. Packard, Phys. Rev. Lett. 83, 3860 (1999).

17. J. Steinhauer, K. Schwab, Yu. M. Mukharsky, J. C. Davis, and R. E. Packard, Physica B 194-196, 767 (1994).

18. R. W. Simmonds, A. Marchenkov, S. Vitale, J. C. Davis, and R. E. Packard, Phys. Rev. Lett. 84, 6062 (2000).

19. A.J. Leggett, Ann. Phys. (New York) 85, 11 (1974).

20. E. V. Thuneberg, J. Kurkijärvi, and J. A. Sauls, Physica B 165\&166, 755 (1990).

21. J. K. Viljas and E. V. Thuneberg, J. Low Temp. Phys. 129, 423 (2002).

22. O. Avenel, Yu. Mukharsky, and E. Varoquaux, Physica $B$ 280, 130 (2000). 